\title{
The role of granulocyte macrophage-colony-stimulating factor in acute intestinal inflammation
}

\author{
Yinghua $\mathrm{Xu}^{1}$, Nicholas $\mathrm{H} \mathrm{Hunt}^{1}$, Shisan $\mathrm{Bao}^{1}$ \\ ${ }^{1}$ Department of Pathology (D06), Bosch Institute, School of Medical Sciences, University of Sydney, New South Wales 2006, \\ Australia
}

An imbalance of mucosal pro- and anti-inflammatory cytokines is crucial in the pathogenesis of inflammatory bowel disease (IBD). GM-CSF influences the development of hemopoietic cells. The precise role of GM-CSF in IBD remains to be elucidated. GM-CSF gene knockout (GM-CSF ${ }^{-/}$) and wild-type (Wt) mice were challenged with $2.5 \%$ dextran sulfate sodium (DSS) for 7 days. The ensued clinical and pathological changes, macrophage infiltration, colonic cytokine production, and bacterial counts were examined. DSS-treated GM-CSF ${ }^{-/-}$mice $^{-}$ developed more severe acute colitis than DSS-treated Wt mice, reflected by a greater body weight loss, more rectal bleeding, and aggravated histopathological changes. More infiltrating macrophages were observed in GM-CSF ${ }^{-/}$, compared with Wt mice following DSS challenge, correlating with monocyte chemoattractant protein-1 (MCP-1) production. The levels of colonic IL-17 and TNF- $\alpha$ were increased significantly in GM-CSF ${ }^{-/}$mice, but not in Wt mice, following DSS administration. The level of IL-6 was increased by 1.5- and 2-fold in the colon of GM-CSF ${ }^{-/}$ and Wt mice, respectively, following DSS challenge. No significant changes in IL-4 and IFN- $\gamma$ were detected in Wt and $\mathrm{GM}_{\mathrm{CSF}}^{-1}$ mice following DSS treatment. The bacteria recovery from colon was increased about 15- and 5-fold, respectively, in Wt mice and $\mathrm{GM}_{-} \mathrm{CSF}^{-/-}$mice following DSS challenge. These results suggest that GM-CSF ${ }^{-/-}$mice are more susceptible to acute DSS-induced colitis, possibly because of an impaired gut innate immune response as a result of diminished GM-CSF.

Keywords: inflammatory bowel disease, GM-CSF, pathogenesis, cytokines, innate immune response Cell Research (2008) 18:1220-1229. doi: 10.1038/cr.2008.310; published online 25 November 2008

\section{Introduction}

GM-CSF is primarily defined as a hematopoietic growth factor that controls the production, differentiation, and function of two related white blood cell populations, the granulocytes and monocyte-macrophages [1, 2]. A major source of GM-CSF is activated T and/or B lymphocytes, with other significant sources being monocytes/macrophages, neutrophils, eosinophils, and epithelial cells [1]. In addition to its physiological role as a hemopoietic growth factor, a growing body of evidence indicates that GM-CSF has effects extending

Correspondence: Shisan Bao

Tel: +61-2-9351-6156; Fax: +61-2-9351-3429

E-mail: bobbao@med.usyd.edu.au

Received 24 December 2007; revised 26 May 2008; accepted 10 June 2008; published online 25 November 2008 well beyond that of its colony-stimulating activity. GMCSF is a highly pleiotropic cytokine with a pronounced effect on many functions of mature granulocytes and macrophages [1-3], including heightening of chemotaxis and cellular adhesion, stimulation of superoxide production and phagocytosis, enhancement of antibodydependent cellular cytotoxicity [4], augmentation of microbicidal capacity, priming production of other proinflammatory cytokines, such as IL-6, IL-8, and TNF- $\alpha$ [5], and inhibition of apoptosis.

GM-CSF is also able to activate and expand antigenpresenting cells through upregulation of MHC class II and $\mathrm{B} 7$ expression $[4,6]$. GM-CSF is critical for the generation, differentiation, and activation of dendritic cells [7], which are recognized as the most important cell type for the stimulation of primary T-cell-mediated immune responses in vivo [8]. GM-CSF is considered to be a potential vaccine adjuvant because of its ability to enhance antigen presentation [9]. 
More recent studies involving GM-CSF gene knockout $\left(\mathrm{GM}_{-} \mathrm{CSF}^{--}\right)$mice indicate that, even though GM-CSF is not required for the maintenance of hematopoiesis in the steady state [10], it is necessary for normal pulmonary homeostasis [11]. Mice lacking GM-CSF have impaired placental formation or function, and reduced long-term survival due to a high incidence of infection [10]. Notably, $\mathrm{GM}^{-\mathrm{CSF}^{-/}}$mice also have significant abnormalities of T-cell function after stimulation by exogenous antigens, evidenced by a delay in $\operatorname{IgG}$ generation, a reduction in IFN- $\gamma$ and IL-4 levels, and an impaired development of cytotoxic CD8 T lymphocyte responses [12].

Inflammatory bowel disease (IBD) is a chronic uncontrolled inflammation of the intestinal mucosa. Despite extensive research, the precise mechanisms underlying IBD remain unclear. A current hypothesis concerning the pathogenesis of IBD is that a dysregulated immune response to intestinal bacterial flora is involved, with both genetic predisposition and environmental factors playing a part [13]. It has become increasingly clear that a disturbed balance of local cytokine production plays a central role in disease progression [14]. Overproduction of pro-inflammatory cytokines, such as IL-6, IL-17, TNF- $\alpha$, IFN- $\gamma$, and GM-CSF, has been reported in many clinical and animal experimental studies $[13,15]$. In addition to the above hypothesis, recently there have been several lines of evidence suggesting that a deficiency of host innate immunity, particularly cellular immunity, may also contribute to the pathogenesis of IBD [16].

Although much progress has been made in understanding the functions of GM-CSF in vitro and in vivo, little is known about the contribution of GM-CSF in IBD. An increased secretion of GM-CSF has been found in mucosal lesions of IBD patients [17], and in a mouse model of colitis induced by dextran sulphate sodium (DSS) [18]. It is believed that mucosal production of GM-CSF is related to a delay in neutrophil apoptosis, which contributes to the tissue injury in IBD [19]. Transgenic GM-CSF expression in the mouse stomach leads to autoimmune gastritis [20]. In contrast to the above findings, some studies indicate that GM-CSF has a protective role in intestinal infection because it can be produced by human intestinal epithelial cells and may regulate the proliferation of intestinal mucosal myeloid cells in response to bacterial invasion [21, 22]. GM-CSF also influences the proliferation of crypt cells in a concentration-dependent manner in vitro [22]. More importantly, a recent clinical trial showed that recombinant human GM-CSF treatment in Crohn's disease led to an improvement, although it did not meet the clinical end-point that was sought [23]. These sometimes contradictory findings about GM-CSF and the mucosa raise interesting questions regarding the precise role of the cytokine in the pathogenesis of IBD. The construction of mice with the GM-CSF gene silenced provides a direct way to investigate the role of this cytokine in the initiation and development of IBD in vivo.

In this study, acute colitis in mice was induced by administering DSS in drinking water for 7 days. To further clarify the role of GM-CSF in the development of intestinal inflammation, we compared the immune and inflammatory responses to acute DSS-induced colitis in ${\mathrm{GM}-\mathrm{CSF}^{-/-}}^{-}$and wild-type (Wt) mice.

\section{Results}

GM-CSF $F^{-/}$mice are more susceptible to acute DSSinduced colitis

None of the unchallenged GM-CSF${ }^{-/}$mice used in this study exhibited the diarrhea, rectal bleeding, and body weight loss that are normally associated with spontaneous intestinal inflammation. Moreover, the histopathological observations did not reveal any evidence of epithelial injury and mucosal inflammation in these mice or in $\mathrm{Wt}$ animals. There was no difference between $\mathrm{Wt}$ and $\mathrm{GM}-\mathrm{CSF}^{-/}$mice in normal drinking water consumption. The body weight decreased progressively in both Wt (Wt-DSS) and GM-CSF ${ }^{-/-}$ (GM-DSS) mice that received $2.5 \%$ DSS treatment. $\mathrm{GM}^{-\mathrm{CSF}^{-/}}$mice started to show clinical signs of illness earlier and more severely than Wt mice following DSS challenge. $\mathrm{GM}-\mathrm{CSF}^{--}$mice had significantly greater $(P$ $<0.05$ ) body weight loss than Wt mice at day 7 of DSS treatment $(83.9 \pm 1.0 \%$ vs $87.7 \pm 0.5 \%$ of baseline body weight, respectively, Figure $1 \mathrm{~A})$. The disease activity index (DAI) based on weight loss, stool consistency, and presence of faecal blood was significantly higher $(P$ $<0.05)$ in $\mathrm{GM}^{-\mathrm{CSF}^{-/}}$mice than in Wt mice at day 7 of DSS treatment (Figure 1B).

No obvious histopathological abnormality was observed in the GM-CSF${ }^{-/}$mice without challenge. Histological damage was seen only in mice with DSS treatment. It was observed that the entire colon, especially the transverse and descending segments, was affected in the DSS-induced colitis model. On histological examination, Wt mice showed less epithelial destruction and inflammatory cell infiltration than did the $\mathrm{GM}^{-\mathrm{CSF}^{-/}}$mice at day 7 of DSS administration (Figure 2A). Histological scores of $4.0 \pm 1.6$ and $6.4 \pm 0.9$ were observed in $\mathrm{Wt}$ and $\mathrm{GM}-\mathrm{CSF}^{-/}$mouse transverse colon, respectively $(P=0.02)$. In the descending colon, histological scores of $3.2 \pm 1.8$ and $5.8 \pm 0.4$ were observed 

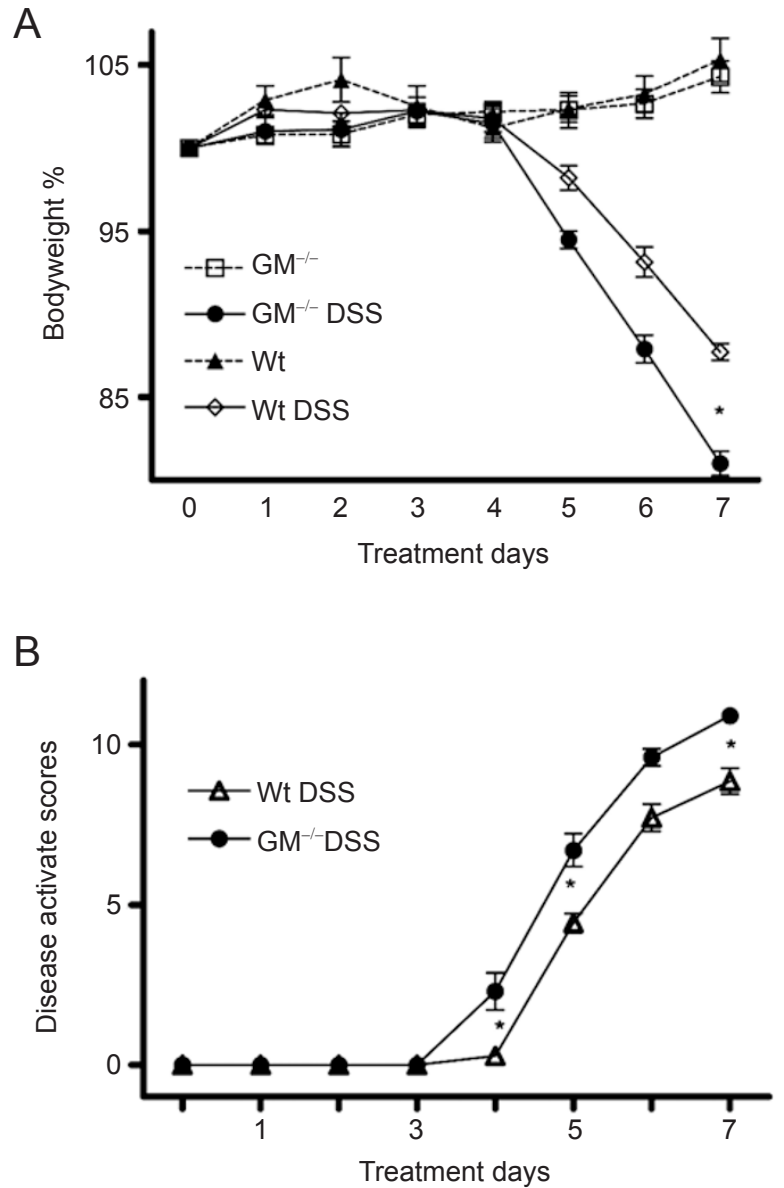

Figure 1 (A) The time course of the body weight loss in $\mathrm{Wt}$ and $\mathrm{GM}^{-\mathrm{CSF}^{-1-}}$ mice given water $\left(\mathrm{Wt}\right.$; $\left.\mathrm{GM}^{--}\right) / \mathrm{DSS}\left(\mathrm{GM}^{-1-} \mathrm{DSS}\right.$; Wt DSS) for 7 days. Data are presented as percentage of original body weight. At day 7 , significantly greater body weight loss was observed in DSS-treated GM-CSF ${ }^{--}$mice compared with the DSS-treated Wt mice. Values are mean \pm SEM for 7-10 mice. ${ }^{*} P<0.05$. (B) The time course of the disease activity index (DAI) in Wt (Wt DSS) and GM-CSF ${ }^{--}\left(\mathrm{GM}^{--}\right.$DSS)mice during DSS treatment. DAI is determined by the body weight loss, presence of fecal blood, and stool consistency, as described in Materials and Methods. A significantly higher DAI $(P=0.03)$ in $\mathrm{GM}^{-\mathrm{CSF}^{-/}}$ mice than in Wt mice was seen at day 7 of DSS treatment. Values are mean \pm SEM for seven mice. ${ }^{*} P<0.05$.

in $\mathrm{Wt}$ and $\mathrm{GM}-\mathrm{CSF}^{-/-}$mice, respectively $(P=0.01$, Figure 2B).

The macroscopic changes associated with DSSinduced colitis classically include shortening of the colon, increase in the ratio of colon weight to length, and splenomegaly [24]. In this study, both DSS-treated Wt and GM-CSF ${ }^{-/}$mice had significantly shorter colons and a higher ratio of colon weight to length than their water-treated littermates (Figure 3A). However, there was no significant difference in colon length and ratio of colon weight to length between Wt and $\mathrm{GM}^{-\mathrm{CSF}^{-/}}$mice treated with DSS (Figure 3A and 3B), or between Wt and $\mathrm{GM}^{-\mathrm{CSF}^{-/}}$mice in the water-treated groups. In general, remarkable increases in the weight of the spleen and the ratio of spleen to initial body weight were seen in DSStreated mice compared with water-treated mice (Figure $3 \mathrm{C})$. Furthermore, GM-CSF${ }^{-/}$mice had a significant increase in spleen weight and percentage of spleen to
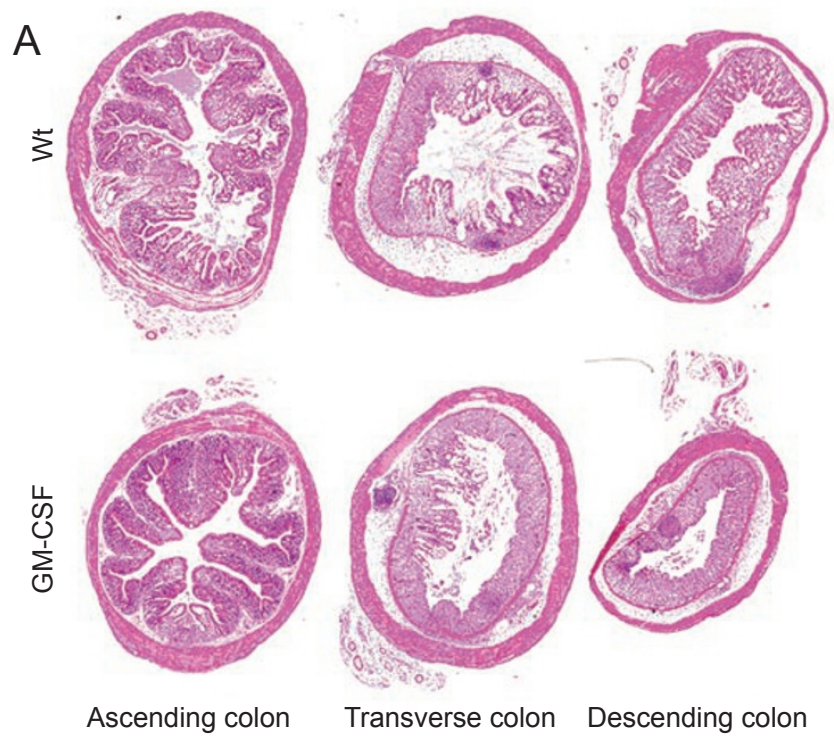

Ascending colon

Transverse colon Descending colon

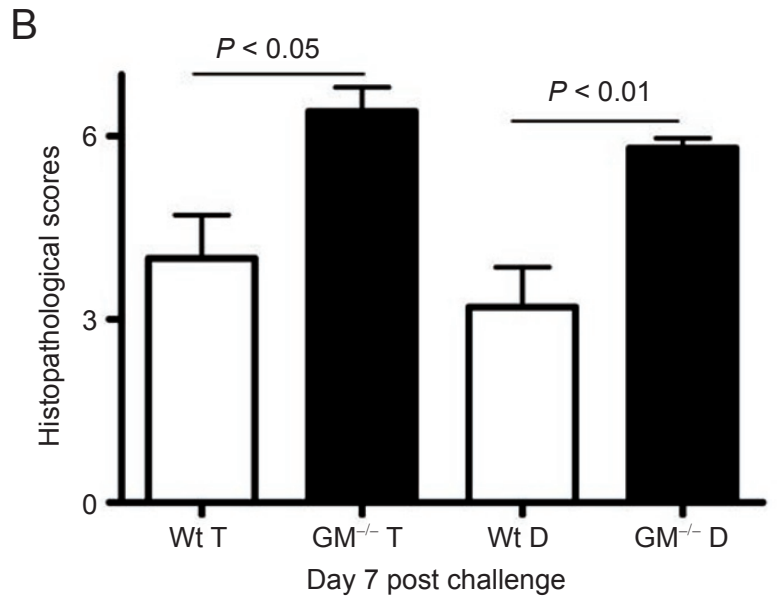

Figure 2 (A) H\&E-stained cross sections of mouse colon from Wt and $\mathrm{GM}^{-\mathrm{CSF}^{--}}$mice after 7 days of DSS treatment. GM$\mathrm{CSF}^{-/}$mice showed more crypt damage and inflammatory cell infiltration than did Wt mice. Photographs were taken under $\times 10$ magnification. (B) Histopathological score of transverse and descending colon from Wt and $\mathrm{GM}_{-} \mathrm{CSF}^{-/-}$mice. $\mathrm{GM}^{-\mathrm{CSF}^{--}}$mice had a significantly higher score in transverse and descending colon than the Wt mice $(P=0.02, P=0.01$, respectively). Values are mean \pm SEM for five mice. T: transverse colon, $D$ : descending colon. 

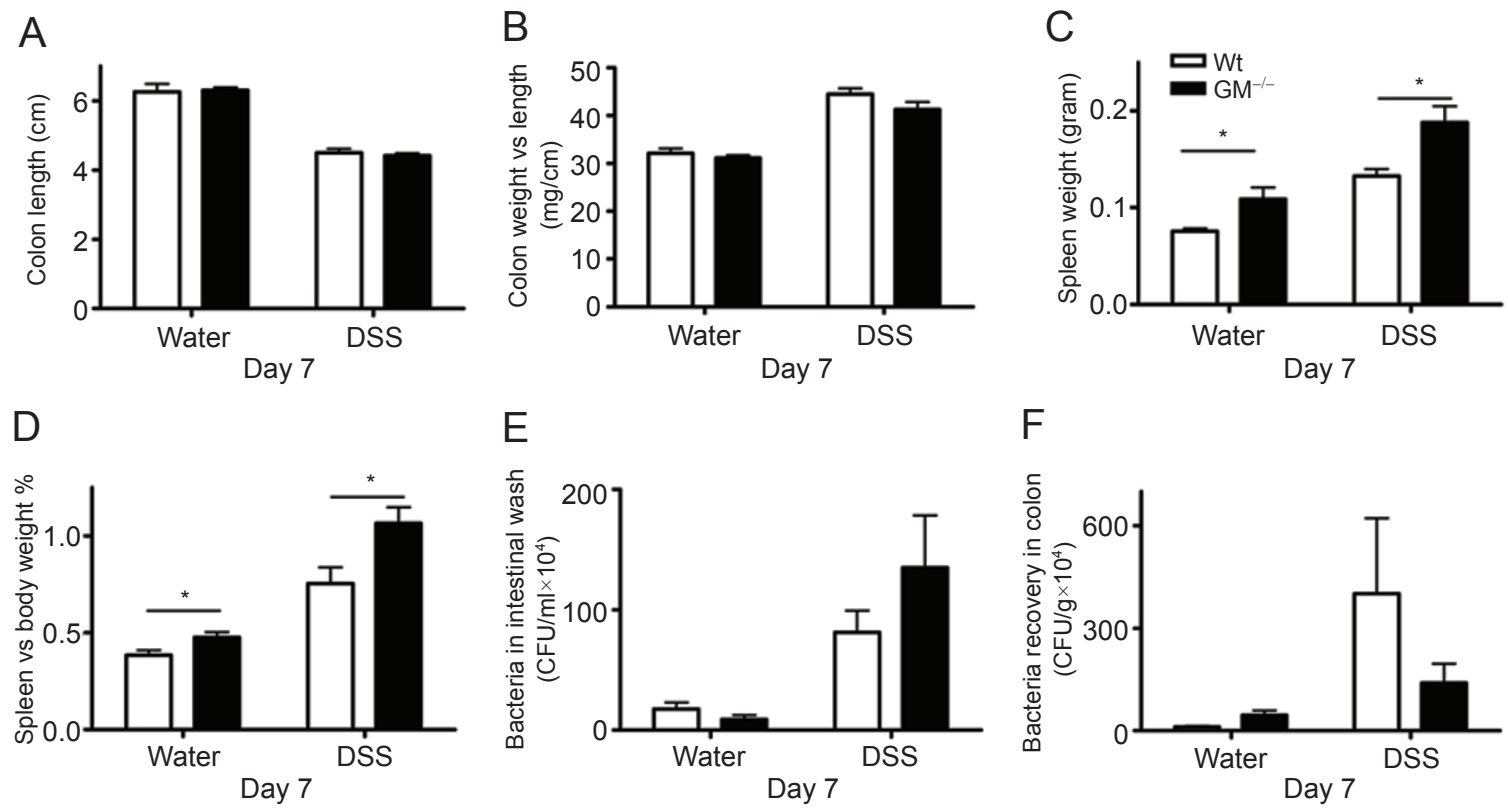

Figure $3 \mathrm{Wt}$ and $\mathrm{GM}^{-\mathrm{CSF}^{--}}$mice colon length (A), colon weight vs length ratio (B), spleen weight (C), spleen vs body weight ratio (D), total bacterial counts in the intestinal washes (E), and fecal pellets (F) on day 7 of DSS treatment. Data are means \pm SEM for 5-7 mice. ${ }^{*} P<0.05$.

body weight when compared with their Wt counterparts, both in the DSS-treated and in the water-treated groups (Figure 3D).

\section{Effects of GM-CSF deficiency on luminal bacteria}

DSS-treated mice had a significantly higher total bacterial count in the intestinal wash than that found in the water-treated mice $(P<0.05)$. DSS-treated GM$\mathrm{CSF}^{-/}$mice displayed an increased total bacterial count in the intestinal wash compared with DSS-treated Wt animals. However, there was no significant difference of total bacterial count between Wt and $\mathrm{GM}-\mathrm{CSF}^{-/-}$ mice in intestinal washes, either with or without DSS treatment. It was noted that in the presence of DSS, the total bacterial count in the intestinal wash was increased almost 12-fold in GM-CSF ${ }^{-/}$mice but only 5-fold in Wt mice compared with their water-treated littermates (Figure 3E). The bacterial recovery from colon was increased about 15- and 5-fold, respectively, in Wt and $\mathrm{GM} \mathrm{CSF}^{--}$mice following DSS challenge (Figure $3 \mathrm{~F})$. However, the number of bacteria that recovered from intestinal wash or the colon was not statistically significantly different between $\mathrm{GM}^{-\mathrm{CSF}^{-/}}$and Wt mice.

\section{Immunohistochemistry}

F4/80 is a commonly used marker of monocytes/ macrophages. In colons of water-treated $\mathrm{Wt}$ and GM$\mathrm{CSF}^{-/}$mice, the $\mathrm{F} 4 / 80^{+}$cells were mainly localized in the lamina propria mucosa, in the subepithelial region, between crypts or at the base of crypts. The submucosa was almost devoid of $\mathrm{F} 4 / 80^{+}$cells in both $\mathrm{GM}-\mathrm{CSF}^{-/}$ mice (Figure 4A-i) and Wt mice (Figure 4A-ii). After 7 days of DSS administration, the numbers of $\mathrm{F} 4 / 80^{+}$cells increased significantly, particularly at the base of crypts and the submucosal layers in both $\mathrm{GM}_{-} \mathrm{CSF}^{-/}$(Figure 4A-iii) and Wt (Figure 4A-iv) mice. Interestingly, DSStreated $\mathrm{GM}-\mathrm{CSF}^{-/}$mice had significantly (almost 2 -fold) more macrophages $(P<0.01)$ in the inflamed tissue than DSS-treated Wt mice (Figure 4B). Moreover, the macrophages appeared to be much smaller, less vacuolated and less foam-like in the colon of DSStreated $\mathrm{GM}-\mathrm{CSF}^{-/-}$mice, i.e. with a relatively less mature appearance, (Figure 4A-iii, inset). The mature macrophages are presented in Figure 4A-iv (inset).

There were few RB6-8C5+ (neutrophil) cells in the colonic tissue of mice treated with tap water. As expected, the numbers of RB6-8C $5^{+}$cells were increased after DSS treatment in both Wt and $\mathrm{GM}_{-} \mathrm{CSF}^{-/-}$mice, localized mainly in the inflamed mucosa and submucosa. Neutrophil infiltration into the submucosal area of the colon in GM-CSF ${ }^{-/}$mice was not significantly different from that in Wt mice (data not shown).

Effects of GM-CSF deficiency on cytokine expression in colon homogenates in acute DSS-induced colitis

The levels of colonic IL-4, IL-6, TNF- $\alpha$, and IL-17 
A

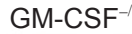
Wt

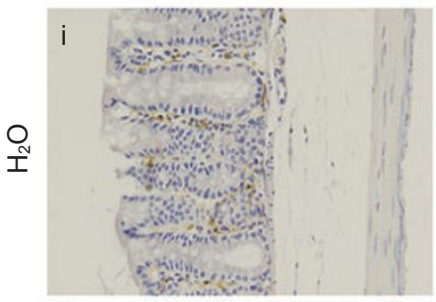

ii
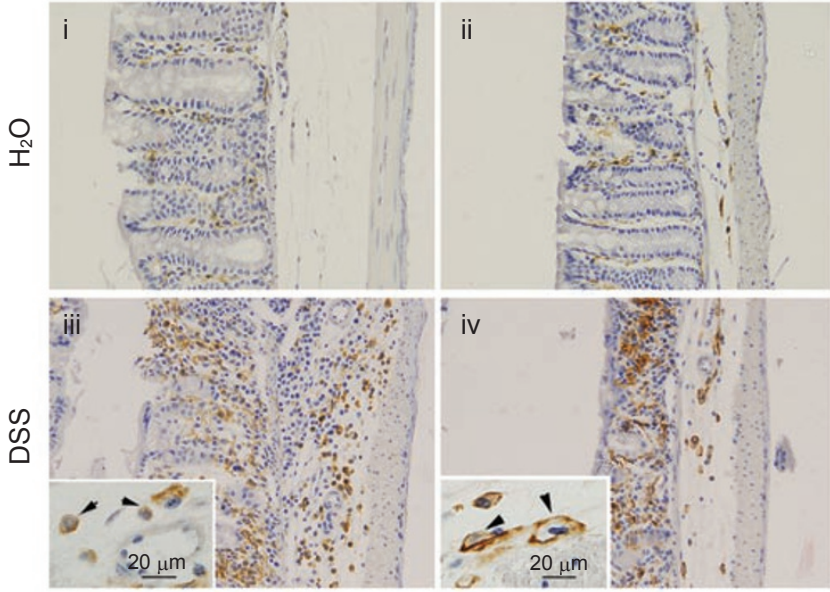

B

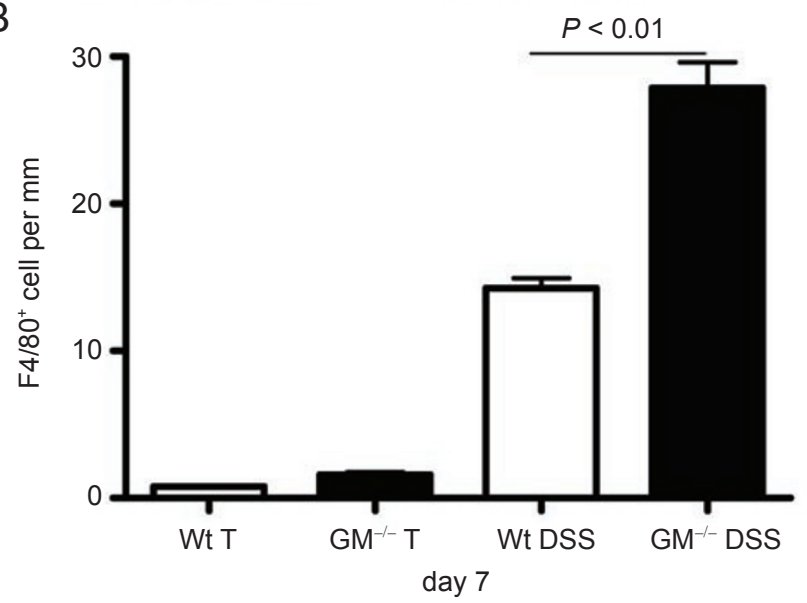

Figure 4 (A) Monocyte/macrophage immunohistostaining in colons of $\mathrm{GM}^{-\mathrm{CSF}^{--}}$(i) and Wt (ii) mice challenged with water only, and $\mathrm{GM}^{-\mathrm{CSF}^{-/}}$(iii) and Wt (iv) mice given DSS treatment. Macrophages were mainly distributed in the gut mucosa of $\mathrm{GM}^{-\mathrm{CSF}^{-l-}}$ (i) and Wt (ii) mice drinking water. The number of macrophages was increased in the gut mucosa and submucosa of GM-CSF${ }^{--}$(i) and Wt (ii) mice with DSS challenge. The macrophages seen in the submucosal area of the colon in GM$\mathrm{CSF}^{-/-}$mice look smaller or less mature (inset) than those in $\mathrm{Wt}$ mice (inset). (B) A significant increase in macrophage infiltration

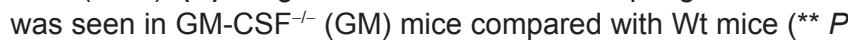
$<0.01)$. Values are mean \pm SEM for five mice.

were significantly lower in water-treated GM-CSF${ }^{-/}$ mice than in their Wt counterparts (Figure 5). After 7 days of DSS treatment, the level of colonic IL-6 was increased by 1.5 - and 2-fold in $\mathrm{GM}-\mathrm{CSF}^{-/}$and $\mathrm{Wt}$ mice, respectively. The levels of colonic IL-17 and TNF- $\alpha$ were significantly increased in $\mathrm{GM}-\mathrm{CSF}^{-/-}$mice, but not in Wt mice. The colonic monocyte chemoattractant protein-1 (MCP-1) level was significantly higher in DSS- treated $\mathrm{GM}^{-\mathrm{CSF}^{-/}}$mice than in DSS-treated Wt mice $(P<0.05)$ (Figure 5A). However, there were no notable changes in the concentrations of colonic IL-4 and IFN- $\gamma$ in Wt and $\mathrm{GM}-\mathrm{CSF}^{-/}$mice following DSS treatment (Figure 5).

\section{Discussion}

Our results show that oral administration of $2.5 \%$ DSS for 7 days induced acute colitis in both Wt and $\mathrm{GM}_{-} \mathrm{CSF}^{-/-}$mice, whereas $\mathrm{GM}-\mathrm{CSF}^{-/-}$mice developed more severe acute colitis than Wt mice. However, GMCSF deficiency per se does not affect the integrity of the normal gut mucosa, and there were no clinical or histological signs of spontaneous colitis. Our current findings in $\mathrm{GM} \mathrm{CSF}^{-/-}$mice are consistent with the report that recombinant GM-CSF ameliorates acute DSS-induced colitis, which has clinical therapeutic implications for treatment of IBD [25].

In this study, deficiency of GM-CSF resulted in an aggravation of DSS-induced colitis in mice, as shown by the three parameters - body weight loss, rectal bleeding, and stool consistency - that comprise the clinical score (Figure 1A and 1B), and by histological observation (Figure 2). A remarkable colon shortening and increase in colon weight to length ratio also were seen in DSStreated $\mathrm{Wt}$ and $\mathrm{GM}-\mathrm{CSF}^{-/-}$mice compared with their water-treated counterparts (Figure 3A and 3B). However, no significant differences were observed between $\mathrm{Wt}$ and $\mathrm{GM}^{-\mathrm{CSF}^{--}}$mice. The body weight reflects the severity of the disease in both the acute and chronic stages, whereas colon length better reflects the severity of the disease in chronic colitis. The greater weight loss in the $\mathrm{GM}^{-\mathrm{CSF}^{-/}}$mice compared with their Wt counterparts, in response to 7 days of DSS challenge, reflects the severity of the acute disease.

In earlier reports no change in spleen size was reported in $\mathrm{GM}_{-} \mathrm{CSF}^{-/}$mice without challenge $[10,11$, 26]. However, in this study, we found that the spleen was enlarged in the water-treated $\mathrm{GM}-\mathrm{CSF}^{-/}$mice (Figure $3 \mathrm{C}$ and 3D). The discrepancy between earlier reports and our current findings suggests that our $\mathrm{GM}-\mathrm{CSF}^{-/-}$mice may have had a subclinical condition. Conceivably, this may have modified host responses, including cytokine changes in both DSS- and water-treated GM-CSF${ }^{-/-}$ mice, and thus impacted on the severity of the colitis. Nevertheless, a higher bacterial load was observed in $\mathrm{GM}_{-\mathrm{CSF}^{-/}}$, compared with $\mathrm{Wt}$, mice following DSS challenge (Figure 3E and 3F). We speculate that the high bacterial load following DSS challenge in the intestine was because of compromised innate immunity resulting in the influx of opportunistic pathogens into 

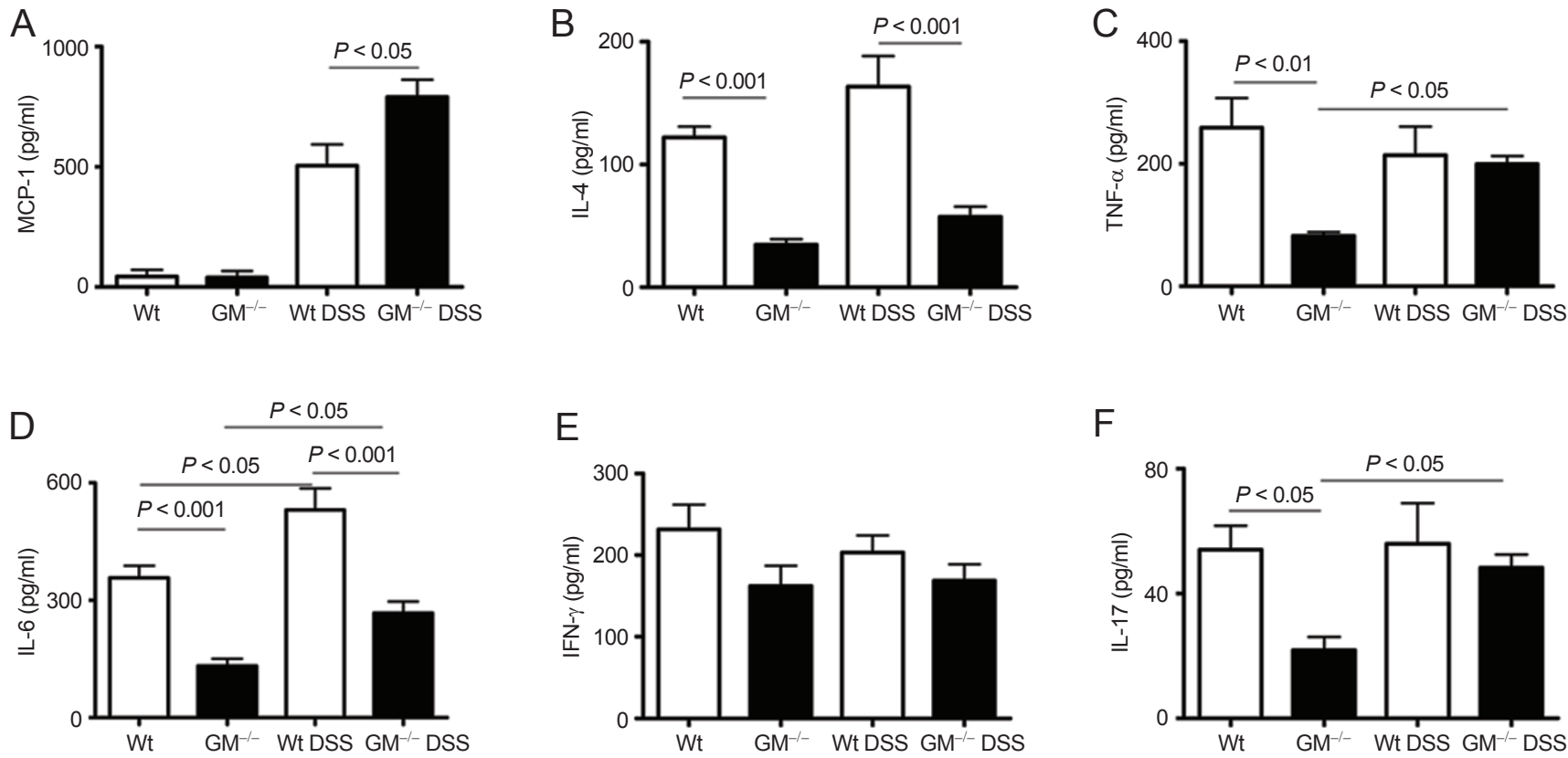

Figure $5 \mathrm{Wt}$ and $\mathrm{GM}_{\mathrm{CSF}}{ }^{--}$mouse colonic inflammatory cytokine and chemokine levels (represented as pg/ml tissue extracts) before and 7 days after DSS treatment. Data are means \pm SEM for 5-7 mice. ${ }^{*} P<0.05,{ }^{* *} P<0.01$.

the lamina propria. This is consistent with findings that lung infections in ${\mathrm{GM}-\mathrm{CSF}^{-/}}^{-}$mice are mainly caused by opportunistic micro-organisms [11].

Clinical IBD and DSS-induced colitis are characterized histologically by local infiltration of neutrophils and macrophages/monocytes. As mentioned, GM-CSF can best be viewed as a major regulator controlling granulocyte and macrophage lineage populations and activation state. However, GM-CSF-deficient mice showed no obvious perturbation in the number of myeloid cells [11]. On the other hand, many studies have shown that, with a sufficiently strong stimulus, GM$\mathrm{CSF}^{-/}$mice cannot sustain the emergency granulocyte and macrophage responses to infection [10, 26, 27]. For these reasons, we assessed neutrophil and macrophage/ monocyte infiltration of colon tissues in this study.

$\mathrm{GM}^{-\mathrm{CSF}^{--}}$mice have been extensively characterized by others and their macrophage lineage pool size is unchanged $[10,11,26]$. Somewhat surprisingly, DSStreated $\mathrm{GM}_{-} \mathrm{CSF}^{-/-}$mice had a remarkable increase in numbers of macrophage/monocytes in colon tissue (Figure 4), but no significant change in the number of neutrophils compared with their Wt counterparts. The macrophages/monocytes in DSS-treated GM-CSF ${ }^{-/}$mice were, however, smaller in size, less vacuolated, and less foam-like (Figure 4A-iii, inset) than in DSS-treated Wt mice (Figure 4A-iv, inset), consistent with less secretion of macrophage-derived pro-inflammatory cytokines (Figure 5) such as IL-6 and TNF- $\alpha$. We speculate that the function of macrophages may be compromised in $\mathrm{GM}-\mathrm{CSF}^{-/}$mice. This is supported by the report from Stanley et al. [11] showing that, in the absence of GMCSF, inflammatory cells can still be localized in tissues such as the lung, although their functional competence may be impaired. This is consistent with the finding that a prominent feature of the lung pathology of $\mathrm{GM}-\mathrm{CSF}^{-/}$ mice is infection with a range of opportunistic bacterial and fungal organisms [11]. The functional differences in infiltrating macrophages between $\mathrm{Wt}$ and gene knockout mice in the intestine will be determined in future studies.

In agreement with earlier studies $[11,28]$, this finding indicates that, in the absence of GM-CSF, inflammatory cells can still be recruited into colonic tissues during the acute stage of DSS-induced colitis, but they may display altered properties consistent with a reduced activation state. Moreover, the significantly increased colonic MCP-1, TNF- $\alpha$, and IL-17 (Figure 5) in DSStreated $\mathrm{GM}_{-} \mathrm{CSF}^{-/-}$mice implies that those inflammatory mediators may substitute for the usual role of GMCSF and contribute to the recruitment of macrophages/ monocytes in this model. Also, of course, compensation from two other CSFs, namely G-CSF and M-CSF, cannot be excluded [29]. All these compensatory effects may result in an overwhelming macrophage response, which 
subsequently leads to more tissue damage and severe intestinal inflammation in $\mathrm{GM}-\mathrm{CSF}^{-/}$mice than in Wt.

The participation of pro-inflammatory cytokines in the pathogenesis of IBD is well recognized [30, 31]. An imbalance of the normal cytokine milieu in the gut might lead to disruption of epithelial function and onset of IBD [1]. It also is well documented that GMCSF has a profound influence on cytokine production by macrophages [32] and T cells [12]. To directly address this issue, we measured cytokine levels in the colon. Under normal conditions, GM-CSF ${ }^{-/}$mice had significantly lower levels of primarily macrophagederived cytokines such as IL- 6 and TNF- $\alpha$, as well as a significant lower level of T-cell cytokines, such as IL-4 and IL-17, in the colon than Wt mice (Figure 5). This result suggests a crucial role of GM-CSF in the activation and function of macrophages/monocytes in the colon in the steady state.

A higher level of MCP-1 and lower levels of IL-4 and IL-6 production in GM-CSF ${ }^{-/-}$mice, compared with Wt mice following DSS challenge, are consistent with earlier findings [33] and might contribute to driving the disease process. As an important cytokine linking innate and adaptive immune responses, GM-CSF deficiency may also affect T-cell proliferation and function under the condition of stimulation [12]. This phenomenon also

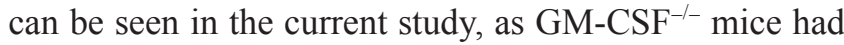
lower anti-inflammatory cytokine (IL-4) and decreased pro-inflammatory cytokine (IFN- $\gamma$ ) levels in the colon after DSS challenge (Figure 5). Earlier reports have revealed that cytokine production in acute DSS-induced colitis is characteristically dominated by macrophagederived cytokines [34]. Consistent with those findings, we did not detect significant changes in colonic IL-4 and IFN- $\gamma$ in either strain of mouse following DSS treatment.

The IL-17, a pro-inflammatory cytokine with a number of functions, plays an important role in IBD. The upregulation of IL-17 at both the mRNA and protein levels has been reported in the intestinal mucosa from IBD patients compared with the non-inflamed normal intestine [35]. Furthermore, IL-17-producing T cells in the gut have been detected in Crohn's disease [36]. A substantial reduction of neutrophil infiltration into the colon was observed in a model of colitis induced by trinitrobenzene sulfonic acid in IL-17-deficient mice [37]. In this study, we found that IL-17 was lower in the colon from $\mathrm{GM}-\mathrm{CSF}^{-/-}$mice compared with Wt mice that received water treatment. However, IL-17 was increased significantly in $\mathrm{GM}-\mathrm{CSF}^{-/}$mice following DSS challenge, but not in Wt mice. The increased IL-17 production is consistent with the more severe colitis in $\mathrm{GM}^{-\mathrm{CSF}^{-/-}}$mice. The precise role of IL-17 in the current acute colitis model remains to be explored, but we speculate that the more severe inflammation in the GM$\mathrm{CSF}^{-/-}$mice was because of a deficiency in macrophage function [11]. The reduced innate immune response may result in a greater influx of intestinal flora into the gut tissue after breakdown of the epithelium following DSS challenge (as shown in Figure 3E and 3F). Subsequently, the higher bacterial load resulted in greater inflammation.

It has been shown that TNF is protective for intestinal inflammation at the acute stage [33]. but is harmful at the chronic stage [38]. This is further supported by findings indicating that the outcomes of anti-TNF treatment for colitis are stage dependent [39]. TNF and IL-6 can be produced by cells other than macrophages. The relationship between intestinal macrophage function and GM-CSF [40] will be investigated further in future.

We have earlier shown that reduced GM-CSF production in $\mathrm{TNF}^{--}$mice correlated with an increased degree of inflammation [33], consistent with our current findings suggesting that GM-CSF provides a protective role in acute gastrointestinal inflammation. The precise mechanism of GM-CSF involvement in the development of colitis is currently being investigated.

The hypothesis that the intestinal bacterial flora contribute to the pathogenesis of IBD is supported by several experimental and clinical observations [40]. It has been reported that bacteria and/or bacterial products play a major role in initiation of acute DSS-induced colitis [41]. Treatment with antibiotics in acute DSSinduced colitis led to significant improvement in clinical and histological parameters [42]. The present results are in agreement with earlier publications showing an increase in total bacterial count in the intestinal wash from DSS-treated mice, although no substantial difference was found between DSS-treated GM-CSF${ }^{-/}$ and $\mathrm{Wt}$ mice. The earlier studies have suggested that different species of commensal enteric bacteria have unequal pro-inflammatory capabilities, with some being more aggressive than others [42, 43]. It is also proposed that different genetic backgrounds have variable dominant bacterial antigenic drives [43]. Thus, GM-CSF deficiency may influence the local colonic microenvironment, favouring the growth of certain bacterial species. More importantly, the alteration of luminal bacterial composition in GM-CSF ${ }^{-/}$mice could possibly contribute to the increased susceptibility of these mice to DSS-induced colitis. It would be of interest to compare the luminal bacterial composition in GM$\mathrm{CSF}^{-/-}$and Wt mice, under normal and inflammatory conditions.

An important and interesting finding from our study is that more severe IBD develops in the GM-CSF${ }^{-/-}$ 
mice. It has been reported that a higher level of GMCSF was observed in the colon from IBD patients, and administration of GM-CSF ameliorated the disease progression [23]. This supports our data showing that mice deficient in GM-CSF developed more severe IBD. These observations may be explained by the role of GM-CSF in the development of IBD being stage dependent, i.e. GM-CSF is beneficial during acute intestinal inflammation, whereas GM-CSF is harmful in chronic inflammation. This parallels the observations on anti-TNF- $\alpha$ treatment, which showed that TNF- $\alpha$ is protective for intestinal inflammation at the acute stage, but is harmful at the chronic stage [44].

In summary, DSS-induced acute colitis was significantly aggravated in $\mathrm{GM}-\mathrm{CSF}^{-/}$mice compared with Wt mice. The mechanisms that cause the enhancement of acute colitis in $\mathrm{GM}^{-\mathrm{CSF}^{-/}}$mice are more likely associated with impaired functions of macrophages (or perhaps dendritic cells), further leading to a dysregulated production of cytokines and chemokines. The alteration of the luminal bacteria composition and delayed crypt regeneration in GM$\mathrm{CSF}^{--}$mice may also play a part. This study provides the first insight into the protective role of GM-CSF in acute mucosal inflammation in vivo. GM-CSF is probably essential for the gut innate immune response, and thus the cytokine could be of benefit for the mice in overcoming bacterial invasion and/or translocation [45] in the early stage of DSS-induced colitis. Nevertheless, both the timing and the duration of GM-CSF expression are important in determining its pathogenic vs protective roles. This information may be useful in optimizing the protocols of GM-CSF treatment of IBD.

\section{Materials and Methods}

\section{Animals \\ GM-CSF-deficient mice (GM-CSF ${ }^{-/-}$mice) on a C57BL/6 $\times 129$ background were generated at the Ludwig Institute for Cancer Research, Melbourne. The GM-CSF ${ }^{-/}$mice were backcrossed

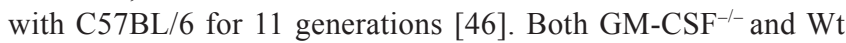 C57BL/6 mice were bred in the University of Sydney, Blackburn Animal House. Age-, sex-, and body weight-matched Wt and GM-CSF ${ }^{-/}$mice were used, and all animals were housed with environmental enrichment in a conventional animal house with free access to food and water. The experiments and procedures were approved by the University of Sydney, Animal Ethics Committee.}

\section{Induction of colitis}

Acute colitis was induced in both GM-CSF${ }^{-/}$and Wt mice by adding 2.5\% (w/v) DSS (ICN Biomedicals, Sydney, Australia) into tap drinking water for seven consecutive days as described earlier [33]. The DSS solutions were freshly prepared daily. The control mice were given only tap water.

\section{Clinical assessment}

Progression of colitis was clinically evaluated daily by measuring drinking volume and body weight, evaluating stool consistency, and by the presence of fecal blood. An earlier validated clinical DAI $[33,47]$ was calculated according to the following parameters: stool consistency (0-4), presence or absence of fecal blood (0-4), and body weight loss (0-4). The maximum possible score is 12 . Colonic inflammation was also evaluated macroscopically by measuring the longitudinal length of the colon from the ileocecal junction to the anal verge, and the colon weight. In addition, colon weight to colon length ratio and the percentage of spleen weight to body weight were calculated.

\section{Histopathological scoring}

Each colon was divided into the proximal, middle, and distal portions, and fixed in cold ethanol as described [48]. Fixed tissues were embedded in paraffin and 5- $\mu \mathrm{m}$-thick sections were stained with H\&E. The mean degree of acute colitis in the middle and distal parts of the colon was calculated from observation of 20 different fields of H\&E-stained longitudinal sections of colon from each animal [49]. A method that modified the earliervalidated histopathological score grading system [50] was used to evaluate the degree of colitis. The crypt distortion, reduction of the proportion of goblet cells, and plasmacytic infiltrates were considered indicative of colonic inflammation. Two independent parameters were measured [51]: the extent of inflammation $(0$, none; 1 , slight; 2 , moderate; 3 , severe; 4 , massive) and the extent of crypt damage ( 0 , none; 1 , the basal one-third portion damaged; 2 , the basal two-thirds portion damaged; 3 , the entire crypt damaged but the surface epithelium intact; 4 , the entire crypt and epithelium lost). The maximum possible score is 8 .

\section{Immunohistochemistry}

The colon, spleen, and mesenteric lymph nodes were fixed in cold ethanol as described [48], embedded in paraffin, and sectioned at $5 \mu \mathrm{m}$. The primary antibodies used in the immunostaining for detection of the macrophage/monocyte marker F4/80 and the granulocyte/neutrophil marker RB6-8C5 were rat monoclonal antibodies to mouse (Rat IgG2a, obtained from The Walter and Eliza Hall Institute of Medical Research, Australia). In brief, sections were incubated with a diluted primary antibody $(1: 200)$ for $1 \mathrm{~h}$ at room temperature. Then the sections were washed three times in tris-buffered saline (TBS), and treated with biotinconjugated secondary antibody for 1 hour at room temperature, followed by avidin-biotin-peroxidase complexes (ABC kit, Vector, Australian Laboratory Service, NSW, Australia). The peroxidase activity was visualized using diaminobenzidine (DAB). The numbers of F4/80-positive cells and Gr-1-positive cells in the submucosa of the transverse colons were counted at high power $(\times 400)$ and averaged in proportion to the length of muscularis mucosa. The infiltrating cells were expressed as the number of cells per millimeter of the muscularis mucosa. An Olympus Bx40 microscope, attached to a digital camera (DP71, Olympus, Sydney, Australia), was used to photograph the slides at $\times 10$ and $\times 40$ magnifications. Images were recorded using a computer-assisted video-imaging system (Olympus DP controller, Sydney, Australia). 


\section{Cytokine ELISA}

The concentrations of IL-4, IL-6, IL-17, TNF- $\alpha$, IFN- $\gamma$, and MCP-1 in supernates of homogenized colon tissue were measured by ELISA using commercial BD OptEIA Set kits (BD Biosciences, Sydney, Australia). The assay was conducted according to the manufacturer's instructions.

\section{Bacterial count}

The samples of intestinal wash were serially diluted 10 -fold in sterile PBS in a sterile tube. Triplicate $10-\mu 1$ drops representing each dilution were plated onto sterile Lura Bertani (LB) agar. The plates were then incubated aerobically at $37{ }^{\circ} \mathrm{C}$ for $24 \mathrm{~h}$ [52]. The colony-forming units (CFU) distributed over each drop zone were counted and averaged for triplicates with countable bacteria from at least two dilutions. The data were expressed as $\mathrm{CFU} / \mathrm{ml}$ intestinal wash.

\section{Statistics}

The experiments were repeated three times, with 5-7 mice per group each time. All data are presented as means \pm SEM. The oneway or two-way ANOVA was performed for statistical analysis. Differences between group means with $P$ values of $<0.05$ were regarded as being statistically significantly different.

\section{Acknowledgments}

This work was supported by a University of Sydney Sesqui grant, Australia. The authors acknowledge assistance from the Histopathology Laboratory, Discipline of Pathology, University of Sydney, Australia.

\section{References}

1 Hamilton JA. GM-CSF in inflammation and autoimmunity. Trends Immunol 2002; 23:403-408.

2 Metcalf D. The granulocyte-macrophage colony-stimulating factors. Science 1985; 229:16-22.

3 Metcalf D. The Florey Lecture, 1991. The colony-stimulating factors: discovery to clinical use. Philos Trans $R$ Soc London Ser B Biol Sci 1991; 333:147-173.

4 Jones TC. The effect of granulocyte-macrophage colony stimulating factor (rGM-CSF) on macrophage function in microbial disease. Med Oncol 1996; 13:141-147.

5 Brissette WH, Baker DA, Stam EJ, Umland JP, Griffiths RJ. GM-CSF rapidly primes mice for enhanced cytokine production in response to LPS and TNF. Cytokine 1995; 7:291-295.

6 Kruger M, Van Gool S, Peng XH, Coorevits L, Casteels-Van Daele M, Ceuppens JL. Production of granulocyte-macrophage colony-stimulating factor by $\mathrm{T}$ cells is regulated by $\mathrm{B} 7$ and IL-1 beta. Immunology 1996; 88:49-54.

7 Storozynsky E, Woodward JG, Frelinger JG, Lord EM. Interleukin-3 and granulocyte-macrophage colony-stimulating factor enhance the generation and function of dendritic cells. Immunology 1999; 97:138-149.

8 Young JW, Steinman RM. Dendritic cells stimulate primary human cytolytic lymphocyte responses in the absence of CD4+ helper T cells. J Exp Med 1990; 171:1315-1332.
9 Pasquini S, Xiang Z, Wang Y, et al. Cytokines and costimulatory molecules as genetic adjuvants. Immunol Cell Biol 1997; 75:397-401.

10 Seymour JF, Lieschke GJ, Grail D, Quilici C, Hodgson G, Dunn AR. Mice lacking both granulocyte colony-stimulating factor (CSF) and granulocyte-macrophage CSF have impaired reproductive capacity, perturbed neonatal granulopoiesis, lung disease, amyloidosis, and reduced long-term survival. Blood 1997; 90:3037-3049.

11 Stanley E, Lieschke GJ, Grail D, et al. Granulocyte/ macrophage colony-stimulating factor-deficient mice show no major perturbation of hematopoiesis but develop a characteristic pulmonary pathology. Proc Natl Acad Sci USA 1994; 91:5592-5596.

12 Wada H, Noguchi Y, Marino MW, Dunn AR, Old LJ. T cell functions in granulocyte/macrophage colony-stimulating factor deficient mice. Proc Natl Acad Sci USA 1997; 94:1255712561.

13 Bouma G, Strober W. The immunological and genetic basis of inflammatory bowel disease. Nat Rev Immunol 2003; 3:521533.

14 Basset C, Holton J. Inflammatory bowel disease: is the intestine a Trojan horse? Sci Progress 2002; 85: 33-56.

15 Strober W, Ludviksson BR, Fuss IJ. The pathogenesis of mucosal inflammation in murine models of inflammatory bowel disease and Crohn disease. Ann Int Med 1998; 128:848856.

16 Moss AC, Farrell RJ. Adding fuel to the fire: GM-CSF for active Crohn's disease. Gastroenterology 2005; 129:21152117.

17 Noguchi M, Hiwatashi N, Liu ZX, Toyota T. Increased secretion of granulocyte-macrophage colony-stimulating factor in mucosal lesions of inflammatory bowel disease. Digestion 2001; 63 Suppl 1:32-36.

18 Dieleman LA, Ridwan BU, Tennyson GS, Beagley KW, Bucy RP, Elson CO. Dextran sulfate sodium-induced colitis occurs in severe combined immunodeficient mice. Gastroenterology 1994; 107:1643-1652.

19 Richter J, Andersson T, Olsson I. Effect of tumor necrosis factor and granulocyte/macrophage colony-stimulating factor on neutrophil degranulation. J Immunol 1989; 142:319931205.

20 Yang YH, Hamilton JA. Dependence of interleukin-1-induced arthritis on granulocyte-macrophage colony-stimulating factor. Arthr Rheumat 2001; 44:111-119.

21 Jung HC, Eckmann L, Yang SK, et al. A distinct array of proinflammatory cytokines is expressed in human colon epithelial cells in response to bacterial invasion. J Clin Invest 1995; 95:55-65.

22 Sennikov SV, Temchura VV, Kozlov VA, Trufakin VA. The influence of conditioned medium from mouse intestinal epithelial cells on the proliferative activity of crypt cells: role of granulocyte-macrophage colony-stimulating factor. $J$ Gastroenterol 2002; 37:1048-1051.

23 Dieckgraefe BK, Korzenik JR. Treatment of active Crohn's disease with recombinant human granulocyte-macrophage colony-stimulating factor. Lancet 2002; 360:1478-1480.

24 Morteau O, Morham SG, Sellon R, et al. Impaired mucosal defense to acute colonic injury in mice lacking 
cyclooxygenase-1 or cyclooxygenase-2. J Clin Invest 2000; 105:469-478.

25 Sainathan SK, Hanna EM, Gong Q, et al. Granulocyte macrophage colony-stimulating factor ameliorates DSSinduced experimental colitis. Inflammatory Bowel Dis 2008; 14:88-99.

26 Paine III R, Preston AM, Wilcoxen S, et al. Granulocytemacrophage colony-stimulating factor in the innate immune response to Pneumocystis carinii pneumonia in mice. $J$ Immunol 2000; 164:2602-2609.

27 Zhan Y, Lieschke GJ, Grail D, Dunn AR, Cheers C. Essential roles for granulocyte-macrophage colony-stimulating factor (GM-CSF) and G-CSF in the sustained hematopoietic response of Listeria monocytogenes-infected mice. Blood 1998; 91:863869.

28 Cook AD, Braine EL, Hamilton JA. Stimulus-dependent requirement for granulocyte-macrophage colony-stimulating factor in inflammation. J Immunol 2004; 173:4643-4651.

29 Hibbs ML, Quilici C, Kountouri N, et al. Mice lacking three myeloid colony-stimulating factors (G-CSF, GM-CSF, and M-CSF) still produce macrophages and granulocytes and mount an inflammatory response in a sterile model of peritonitis. J Immunol 2007; 178:6435-6443.

30 Murai M, Yoneyama H, Harada A, et al. Active participation of CCR5(+)CD8(+) T lymphocytes in the pathogenesis of liver injury in graft-versus-host disease. J Clin Invest 1999; 104:4957.

31 Monteleone I, Vavassori P, Biancone L, Monteleone G, Pallone F. Immunoregulation in the gut: success and failures in human disease. Gut 2002; 50 Suppl 3:III60-III64.

32 Borrello I, Pardoll D. GM-CSF-based cellular vaccines: a review of the clinical experience. Cytokine Growth Factor Rev 2002; 13:185-193.

$33 \mathrm{Xu}$ Y, Hunt NH, Bao S. The correlation between proinflammatory cytokines, MAdCAM-1 and cellular infiltration in the inflamed colon from TNF- $\alpha$ gene knockout mice. Immunol Cell Biol 2007; 85:633-639.

34 Dieleman LA, Palmen MJ, Akol H, et al. Chronic experimental colitis induced by dextran sulphate sodium (DSS) is characterized by Th1 and Th2 cytokines. Clin Exp Immunol 1998; 114:385-391.

35 Fujino S, Andoh A, Bamba S, et al. Increased expression of interleukin 17 in inflammatory bowel disease. Gut 2003; 52:65-70.

36 Annunziato F, Cosmi L, Santarlasci V, et al. Phenotypic and functional features of human Th17 cells. J Exp Med 2007; 204:1849-1861.

37 Zhang Z, Zheng M, Bindas J, Schwarzenberger P, Kolls JK. Critical role of IL-17 receptor signaling in acute TNBSinduced colitis. Inflammatory Bowel Dis 2006; 12:382-388.

38 Kato S, Hokari R, Matsuzaki K, et al. Amelioration of murine experimental colitis by inhibition of mucosal addressin cell adhesion molecule-1. J Pharmacol Exp Ther 2000; 295:183189.

39 Kamada N, Hisamatsu T, Okamoto S, et al. Abnormally differentiated subsets of intestinal macrophage play a key role in Th1-dominant chronic colitis through excess production of IL-12 and IL-23 in response to bacteria. J Immunol 2005; 175:6900-6908

40 Philpott DJ, Girardin SE, Sansonetti PJ. Innate immune responses of epithelial cells following infection with bacterial pathogens. Cur Opin Immunol 2001; 13:410-416.

41 Hernandez GA, Appleyard CB. Bacterial load in animal models of acute and chronic 'reactivated' colitis. Digestion 2003; 67:161-169.

42 Rath HC, Schultz M, Freitag R, et al. Different subsets of enteric bacteria induce and perpetuate experimental colitis in rats and mice. Infect Immun 2001; 69:2277-2285.

43 Sartor RB. The influence of normal microbial flora on the development of chronic mucosal inflammation. Res Immunol 1997; 148:567-576.

44 Kojouharoff G, Hans W, Obermeier F, et al. Neutralization of tumour necrosis factor (TNF) but not of IL-1 reduces inflammation in chronic dextran sulphate sodium-induced colitis in mice. Clin Exp Immunol 1997; 107:353-358.

45 Gennari R, Alexander JW, Gianotti L, Eaves-Pyles T, Hartmann S. Granulocyte macrophage colony-stimulating factor improves survival in two models of gut-derived sepsis by improving gut barrier function and modulating bacterial clearance. Ann Surg 1994; 220:68-76.

46 Campbell IK, Rich MJ, Bischof RJ, Dunn AR, Grail D, Hamilton JA. Protection from collagen-induced arthritis in granulocyte-macrophage colony-stimulating factor-deficient mice. J Immunol 1998; 161:3639-3644.

47 Cooper HS, Murthy SN, Shah RS, Sedergran DJ. Clinicopathologic study of dextran sulfate sodium experimental murine colitis. Lab Invest 1993; 69:238-249.

48 Bao S, Beagley KW, France MP, Shen J, Husband AJ. Interferon-gamma plays a critical role in intestinal immunity against Salmonella typhimurium infection. Immunology 2000; 99:464-472.

49 Mackay F, Browning JL, Lawton P, et al. Both the lymphotoxin and tumor necrosis factor pathways are involved in experimental murine models of colitis. Gastroenterology 1998; 115:1464-1475.

50 Wang L, Walia B, Evans J, Gewirtz AT, Merlin D, Sitaraman SV. IL-6 induces NF-kappa B activation in the intestinal epithelia. J Immunol 2003; 171:3194-3201.

51 Kabashima K, Saji T, Murata T, et al. The prostaglandin receptor EP4 suppresses colitis, mucosal damage and CD4 cell activation in the gut. J Clin Invest 2002; 109:883-893.

52 Hochepied T, Wullaert A, Berger FG, et al. Overexpression of alpha(1)-acid glycoprotein in transgenic mice leads to sensitisation to acute colitis. Gut 2002; 51:398-404. 